\title{
Proteomic landscape of human coronary artery atherosclerosis
}

\author{
YAQING ZHOU ${ }^{1 *}$, JINXIA YUAN $^{1 *}$, YINWEN FAN $^{2}$, FENGHUI AN $^{2}$, JIAXIN CHEN $^{1}$, \\ YONGJIE ZHANG ${ }^{3}$, JIANLIANG JIN ${ }^{3}$, MUFENG GU ${ }^{3}$, ZHIYUAN MAO ${ }^{3}$, HAIJIAN SUN ${ }^{3}$, QIAOWEI JIA ${ }^{1}$, \\ CHENHUI ZHAO $^{1}$, MINGYUE JI ${ }^{1,4}$, JING ZHANG ${ }^{1}$, GUANGXU XU ${ }^{5}$ and ENZHI JIA ${ }^{1}$ \\ ${ }^{1}$ Department of Cardiovascular Medicine, The First Affiliated Hospital of Nanjing Medical University, Nanjing, \\ Jiangsu 210029; ${ }^{2}$ Department of Cardiovascular Medicine, The Friendship Hospital of Ili Kazakh Autonomous Prefecture, \\ Yining, Xinjiang 835000; ${ }^{3}$ Department of Human Anatomy, Nanjing Medical University, Nanjing, Jiangsu 210029; \\ ${ }^{4}$ Department of Cardiovascular Medicine, Lianshui People's Hospital, Lianshui, Jiangsu 223400; \\ ${ }^{5}$ Department of Rehabilitation Medicine, The First Affiliated Hospital of Nanjing Medical University, \\ Nanjing, Jiangsu 210029, P.R. China
}

Received November 4, 2019; Accepted April 24, 2020

DOI: $10.3892 /$ ijmm.2020.4600

\begin{abstract}
In order to investigate novel biomarkers for the detection of coronary artery disease for effective therapeutic targets, a comprehensive understanding of the protein networks and protein expression abundance in coronary artery samples is required. This was established by means of liquid chromatography (LC)-mass spectrometry (MS)/MS analysis in the present study. A total of 20 human coronary artery specimens from 2 autopsied adults were employed in the present study. The natural history and histological classification of the atherosclerotic lesions of the coronary artery samples were analyzed by hematoxylin and eosin (H\&E) staining, and the human coronary arterial proteome and proteomics features were characterized by MS analysis. The present study identified 2,135 proteins in the 20 coronary artery segments samples from the 2 cases. Combined with the results of H\&E staining of the coronary artery samples, a total of 174 proteins, including 4 upregulated proteins and 164 downregulated proteins (excluding 6 proteins with inconsistent expression tendencies), were shown to be associated with coronary artery disease. In addition, Gene Ontology and Kyoto Encyclopedia of Genes and Genomes enrichment of the differentially expressed proteins revealed that the mitochondrial energy metabolism may be responsible for the occurrence and development of coronary artery atherosclerosis. The human coronary arterial
\end{abstract}

Correspondence to: Dr Enzhi Jia, Department of Cardiovascular Medicine, The First Affiliated Hospital of Nanjing Medical University, 300 Guangzhou Road, Nanjing, Jiangsu 210029, P.R. China

E-mail: enzhijia@njmu.edu.cn

${ }^{*}$ Contributed equally

Key words: coronary artery, coronary artery disease, proteomics, mitochondrion, hematoxylin and eosin staining proteome can be considered as a complex network whose architectural characteristics vary considerably as a function of the presence or absence, and histological classification of coronary artery atherosclerosis. These data thus suggest that the prevention of mitochondrial dysfunction via the retrieval of the mitochondrial associated proteins expression may be a promising target in coronary artery disease.

\section{Introduction}

Recently, as one of the most dominant platforms to spatiotemporally resolve protein interaction networks in living cells (1), refine existing protein-protein interaction networks (2), identify potential therapeutic target (3) and distinguish new protein therapeutics (4), mass spectrometry (MS)-based proteomics is characterized by its ability to present detailed information of protein intensities. It can thus provide a more comprehensive landscape of cellular process and enable network-centered investigations (5). Therefore, MS-based proteomics has emerged as a dominant tool in the field of biomarker investigations for various diseases. Due to the distinguishing characteristics, label-free proteome quantification (LFQ) has become increasingly investigated, and has been widely applied to the protein biomarker investigations for coronary artery disease.

According to its molecular biology, coronary artery disease can be defined as a group of thousands of proteins that collectively alter cellular processes and result in the characteristic remodeling of the local coronary artery environment. Therefore, to achieve the early diagnosis of coronary artery disease, and to interfere with the coronary artery disease process before clinical consequences appear, it is essential to identify the unique patterns and dynamic characteristics of coronary arterial protein networks that consist of the molecular signatures of normal and atherosclerotic coronary arterial tissues.

Previous studies have presented characteristics of the human coronary arterial proteome in autopsied young adults (6), and paraformaldehyde-fixed, paraffin-embedded and frozen coronary arteries (7); limited numbers of global human coronary 
arterial tree samples with various grade atherosclerosis have been investigated. To the best of our knowledge, comprehensive studies on the proteins associated with coronary artery disease have not yet been published. Therefore, a label-free proteome quantification technology-based landscape study of the proteomics characteristics of human coronary atherosclerosis was performed in the present study, in order to characterize human coronary arterial proteomics, and to investigate the proteins, networks and pathways most significantly associated with human coronary arterial atherosclerotic lesions.

\section{Subjects and methods}

Study subjects. The coronary artery samples were obtained from 2 autopsy cases from the Department of Human Anatomy in Nanjing Medical University. Informed consent from the bereaved family was obtained for the research use only of the samples and the autopsy was performed according to the guidelines of the university. The methods were performed in accordance with the approved guidelines, and all experimental protocols were approved by the Ethics Committee of Nanjing Medical University and the First Affiliated Hospital of Nanjing Medical University. The experimental design and schematic roadmap of the present study is presented in Fig. 1.

Preparation of coronary artery segments. The present study included 2 autopsies performed in 2018 at the Department of Human Anatomy at Nanjing Medical University. The age of the patients was 64 and 69 years, and both were male. The postmortem delay varied between 1 and 2 days. During the autopsy, epicardial coronary arteries were removed from the hearts. The epicardial coronary artery of each autopsy was divided into 10 segments as follows: The proximal segment, midsegment and distal segment of the left anterior descending (LAD), proximal segment, midsegment and distal segment of the left circumflex (LCX), proximal segment, midsegment and distal segment of the right coronary artery (RCA) and the left main trunk (LM). Each coronary artery segment was divided into 2 groups as follows: The protein group and the pathological group. The segments at a thickness of $5 \mathrm{~mm}$ in the protein group were snap-frozen in liquid nitrogen and stored at $-80^{\circ} \mathrm{C}$ for protein extraction. In addition, the segments at a thickness of $5 \mathrm{~mm}$ in the pathological group were fixed overnight in $10 \%$ formalin and embedded in paraffin for histological analysis following decalcification in ethylene diamine tetraacetic acid (EDTA) decalcification fluid (Beijing Solarbio Science \& Technology Co., Ltd.) for approximately 2 weeks.

Pathological analysis. Following decalcification with EDTA decalcification fluid (Beijing Solarbio Science \& Technology Co., Ltd.) for $\sim 2$ weeks, the coronary artery segments in the pathological group were fixed overnight in $10 \%$ formalin and processed for paraffin embedding: Longitudinal 5- $\mu \mathrm{m}$-thick consecutive sections were obtained using a rotary microtome (Leica RM2235; Leica Biosystems Nussloch GmbH) and stained with hematoxylin and eosin (H\&E) in order to observe the morphology. Each slide was examined using a steromicroscope 10X (Leica DM2500; Leica Microsystems) at x5 and $\times 20$ magnification and digitized using an image analysis system (Leica LAS; Leica Microsystems).
The coronary atherosclerosis grade and extent of the coronary artery segments were analyzed by independent pathologists who graded the samples based on the American Heart Association (AHA) classification guidelines (8).

\section{Protein extraction and LC-MS/MS analysis}

Coronary artery segment. A total of $100 \mathrm{mg}$ of each coronary artery sample was obtained for the extraction experiment. Coronary artery samples were ground in liquid nitrogen and transferred to a centrifuge tube. A total of $600 \mu \mathrm{l}$ of L3 lysis buffer (7 M urea, $2 \mathrm{M}$ thiourea, $40 \mathrm{mM}$ Tris-HCl) was added to make a final concentration with $1 \mathrm{mM}$ phenylmethanesulfonyl fluoride (PMSF), $2 \mathrm{mM}$ EDTA and $10 \mathrm{mM}$ DL-Dithiothreitol (DTT). The solution was mixed with a pipette and ultrasonically treated on ice for $15 \mathrm{~min}$ $\left(2 \mathrm{sec} / 3 \mathrm{sec}\right.$ ); and then centrifuged at $20,000 \mathrm{x} \mathrm{g}$ at $4^{\circ} \mathrm{C}$ for $20 \mathrm{~min}$. The supernatant was then added to 4 times the volume of cold acetone, yielding a final concentration up to $30 \mathrm{mM}$ DTT. This solution was precipitated at $-20^{\circ} \mathrm{C}$ for $2 \mathrm{~h}$; it was then centrifuged at $20,000 \mathrm{xg}$ at $4^{\circ} \mathrm{C}$ for $20 \mathrm{~min}$. The supernatant was discarded, and $1 \mathrm{ml}$ of cold acetone was added to the precipitate, yielding a final concentration of $10 \mathrm{mM}$ DTT. The precipitate was completely crushed, vortexed, allowed to stand at $-20^{\circ} \mathrm{C}$ for $30 \mathrm{~min}$, centrifuged at $20,000 \mathrm{x} \mathrm{g}$ for $20 \mathrm{~min}$, and the supernatant was then discarded. Subsequently, 100-600 $\mu 1$ U2 lysis buffer (8 M Urea, 10 mM EDTA) were added, and the solution was ultrasonically treated on ice for $5 \mathrm{~min}(2 \mathrm{sec} / 3 \mathrm{sec})$, and then centrifuged at $20,000 \mathrm{x} \mathrm{g}$ for $20 \mathrm{~min}$ at $4^{\circ} \mathrm{C}$. The supernatant was then removed. The protein concentration was determined using the Bradford method.

Protein quantification. A total of $2 \mu \mathrm{l}$ of the protein sample was obtained, diluted to the appropriate amount, and the protein concentration was subsequently determined using the Bradford method as follows: A total of $0.02 \mu \mathrm{g} / \mu \mathrm{l}$ of bovine serum albumin (BSA) was configured to a standard $2 \mathrm{ml}$. Subsequently, 0, 20, 40, 60, 80, 100, 120, 140, 160, 180 and $200 \mu \mathrm{l}$ were added to a new $1.5 \mathrm{ml} \mathrm{EP}$ tube, and the diluent was added. Up to $200 \mu \mathrm{l}$ was added and mixed. Subsequently, $20 \mu \mathrm{l}$ of the diluted BSA solution were placed into the microplate, with 3 replicate wells for each concentration. A total of $20 \mu$ l of the diluted protein sample was added to the plate, and 3 replicate wells were set for each sample. This was followed by the addition of $180 \mu \mathrm{l}$ of G250 color solution to each well and incubation for $5 \mathrm{~min}$ at room temperature. The A595 absorbance value was measured with a microplate reader (SM600, Shanghai Utrao Medical Instrument Co., Ltd.). The concentration of the protein sample was calculated from the standard curve and the dilution factor of the protein sample.

Reductive alkylation. For each coronary artery sample, $500 \mu \mathrm{g}$ of total protein were added to $200 \mu 1 \mathrm{U} 2$ lysis buffer, with $1 \%$ of the volume of $1 \mathrm{M} \mathrm{DTT}$, kept in a water bath at $56^{\circ} \mathrm{C}$ for $30 \mathrm{~min}$, and the temperature was then rapidly returned to room temperature by placing the solution on ice. Subsequently, $10 \%$ of the volume of $0.55 \mathrm{M}$ iodoacetamide (IAM) was established, and kept in the dark at room temperature for $30 \mathrm{~min}$. A total of 4 times the volume of cold acetone was then added, as well as the final concentration of $10 \mathrm{mM}$ DTT, and the samples were then precipitated at $-20^{\circ} \mathrm{C}$ for $2 \mathrm{~h}$. The samples were then centrifuged at $2,000 \mathrm{x} \mathrm{g}$ for $20 \mathrm{~min}$ at $4^{\circ} \mathrm{C}$, the supernatant was discarded and $1 \mathrm{ml}$ of cold acetone was added, as well as 
A

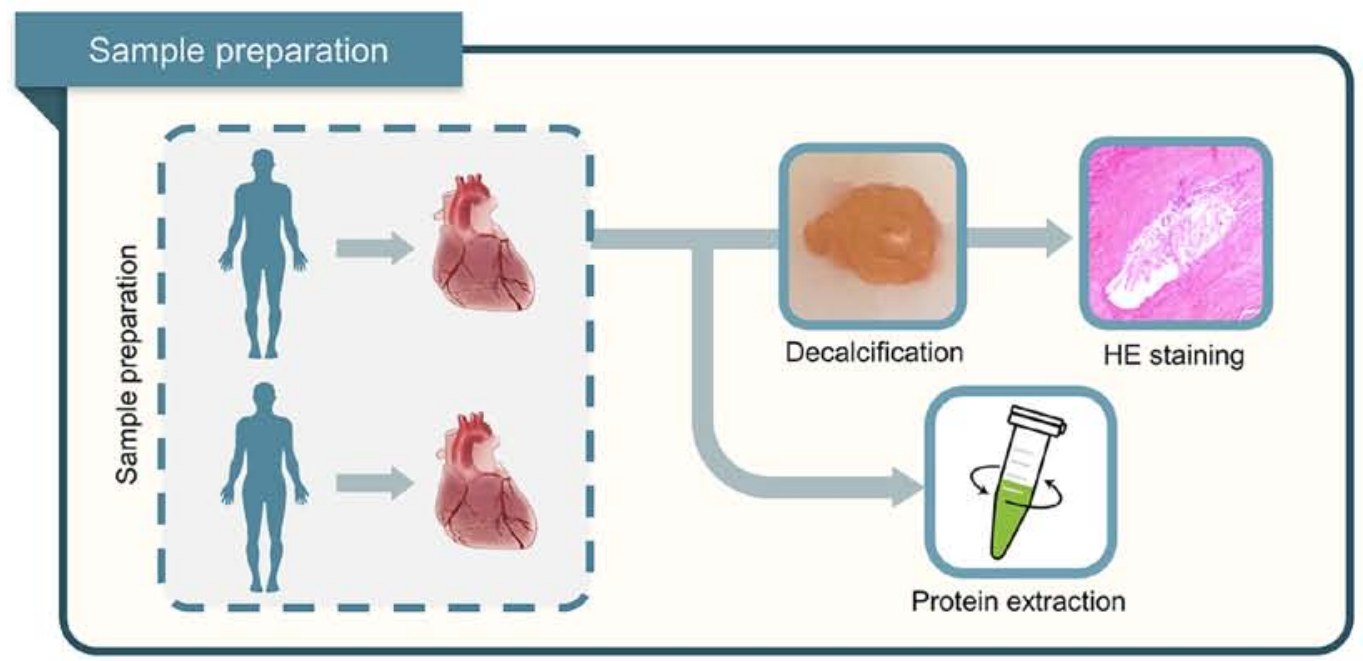

B

\section{LC-MS/MS analysis}

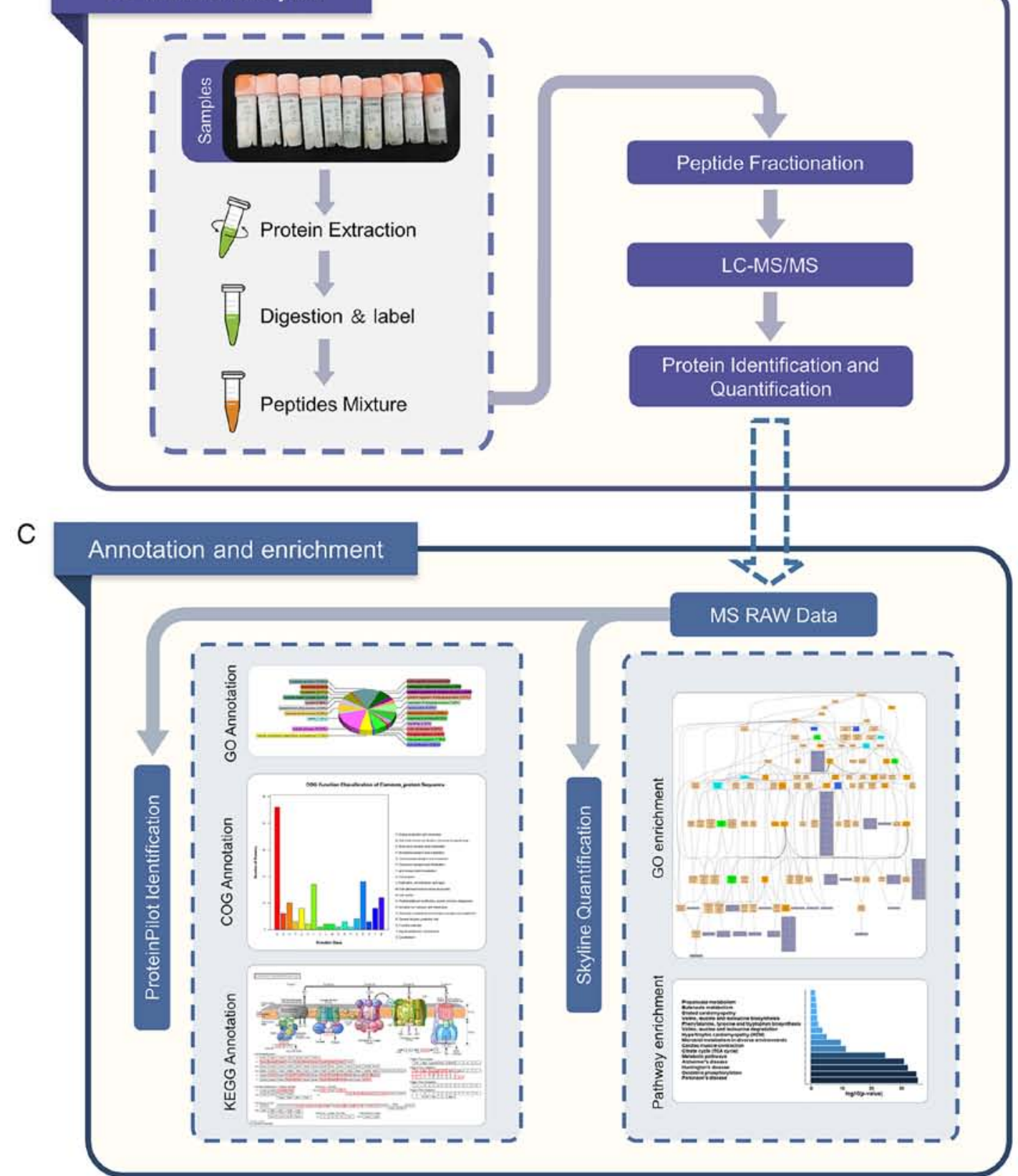

Figure 1. Overall technological schematic of the present study. (A) Coronary artery sample preparation. (B) LC-MS/MS analysis. (C) Bioinformatics analysis. LC-MS/MS, liquid chromatography-mass spectrometry. 
DTT at the final concentration of $10 \mathrm{mM}$. The precipitate was mashed, vortexed, lapsed at $-20^{\circ} \mathrm{C}$ for $30 \mathrm{~min}$, and centrifuged at 20,000 x g for $20 \mathrm{~min}$, before discarding the supernatant. The precipitate was air-dried, $300 \mu 1 \mathrm{U} 2$ lysis buffer were added, sonicated for $5 \mathrm{~min}(2 \mathrm{sec} / 3 \mathrm{sec})$ and centrifuged at 20,000 x g for $20 \mathrm{~min}$ at $4^{\circ} \mathrm{C}$. The supernatant was obtained, and the protein concentration was determined via the Bradford method.

Protein digestion. The supernatant from each sample, containing precisely $100 \mu \mathrm{g}$ of protein was digested with Trypsin Gold (Promega) at a 1:50 enzyme-to-substrate ratio. After $16 \mathrm{~h}$ of digestion at $37^{\circ} \mathrm{C}$, peptides were desalted with $\mathrm{C} 18$ cartridge to remove the high urea, and desalted peptides were dried by vacuum centrifugation at $4^{\circ} \mathrm{C}$ and $1,000 \mathrm{x}$ for $3 \mathrm{~h}$.

$L C-M S / M S$ analysis. All protein samples were analyzed using Q Exactive mass spectrometer (Thermo Fisher Scientific, Inc.) coupled with the Easy-nLC 1200 UHPLC system (Thermo Fisher Scientific, Inc.). A sample volume containing $2 \mu \mathrm{g}$ of total peptides was injected onto a home-made $\mathrm{C} 18$ trap column $(5 \mu \mathrm{m}, 100 \mu \mathrm{m} \times 20 \mathrm{~mm})$, and eluted at $300 \mathrm{nl} / \mathrm{min}$ onto a C18 analytical column ( $3 \mu \mathrm{m}, 75 \mu \mathrm{m} \times 150 \mathrm{~mm})$ with a 120 min gradient. A binary mobile phase system of buffer A $\left(2 \%\right.$ acetonitrile/ $0.1 \%$ formic acid/98\% $\left.\mathrm{H}_{2} \mathrm{O}\right)$ and buffer $\mathrm{B}$ (98\% acetonitrile/ $0.1 \%$ formic acid/2\% $\mathrm{H}_{2} \mathrm{O}$ ) was used. The $120 \mathrm{~min}$ solvent gradient listed as follows: $5 \% \mathrm{~B}, 5 \mathrm{~min}$; 5-25\% B, $85 \mathrm{~min} ; 25-35 \% \mathrm{~B}, 10 \mathrm{~min} ; 35-80 \% \mathrm{~B}, 10 \mathrm{~min}$; $80 \%$ B, 5 min; $80-5 \%, 1 \mathrm{~min} ; 5 \%, 4 \mathrm{~min}$. The LC was interfaced to a Q-Exactive quadrupole Orbitrap mass spectrometer (Thermo Fisher Scientific, Inc.) via nano-electrospray ionization using an Easy Spray source with an integrated column heater set at $50^{\circ} \mathrm{C}$. An electrospray voltage of $2.2 \mathrm{kV}$ was applied. The mass spectrometer was programmed to acquire, by data-dependent acquisition, tandem mass spectra from the top 20 ions in the full scan from 400 to $1200 \mathrm{~m} / \mathrm{z}$. Dynamic exclusion was set to $15 \mathrm{sec}$, singly-charged ions were excluded, isolation width was set to 1.6 Da, full MS resolution to 70,000 and MS/MS resolution to 17,500 . Normalized collision energy was set to 25, automatic gain control to 1e6, max fill of MS to $20 \mathrm{msec}$, max fill MS/MS to $60 \mathrm{msec}$, and the underfill ratio to $0.1 \%$.

Processing of mass spectrometry spectra by MaxQuant. The resulting MS/MS data were processed using Maxquant search engine (v.1.5.2.8). Tandem mass spectra were searched against the UniProt Homo_sapiens protein database (https://www. uniprot.org/) concatenated with the reverse decoy database. Trypsin/P was specified as cleavage enzyme allowing up to 4 missing cleavages. The mass tolerance for precursor ions was set as 20 ppm in First search and 5 ppm in Main search, and the mass tolerance for fragment ions was set as $0.02 \mathrm{Da}$. The carbamidomethylation of cysteine was considered as a fixed modification and oxidation of methionine, acetylation of the $\mathrm{N}$-terminus, and the deamidation of asparagine or glutamine as variable modifications. The label-free quantification method was LFQ, FDR was adjusted to $<1 \%$ and the minimum score for modified peptides was set $>40$.

For protein identification, protein with at least 1 unique peptide was identified at FDR $<1.0 \%$ on the peptide and protein level, respectively. Proteins containing similar peptides and that could not be distinguished based on MS/MS analysis were grouped separately as protein groups. Precursor quantification based on intensity was used for label-free quantification. The significant ratios, defined as fold change (FC) $\geq 1.5$ or FC $\leq 0.67$, were used to screen the differentially expressed proteins (DEPs) (9).

Bioinformatics and annotations. In order to determine the biological and functional properties of all identified proteins, the Gene Ontology (GO) Terms (http://geneontology.org/) were used to describe the properties of genes and gene products in organisms. To this end, homology searches were first performed on all identified sequences using the local NCBI blastp program for the NCBInr animal database in the present study. The e value was set to be $<1 \mathrm{e}-5$, and the best hit for each query sequence takes into account the GO term match. GO term matching was performed using blast2go v4.5. Functional annotation of genes from the new genome and studies of genome evolution were investigated using the orthologous protein system population [Clusters of Orthologous Groups of proteins (COGs) database; http://www.ncbi.nlm.nih. gov/COG/)]. The Kyoto Encyclopedia of Genes and Genomes (KEGG) is the main public database on pathways (https://www. kegg.jp/), and pathway analyses identify the most important biochemical metabolic pathways and signal transduction pathways involved with each protein. In order to identify candidate biomarkers, the present study used hypergeometric testing for GO enrichment and KEGG pathway enrichment.

\section{Results}

Natural history and histological classification of atherosclerotic lesions of the coronary artery samples. The natural history and histological classification of atherosclerotic lesions of the coronary artery samples were analyzed by H\&E staining in the present study, and the results are presented in Table I and Figs. 2 and 3.

The analysis of the H\&E staining indicated that the atherosclerotic lesions were observed in the coronary artery segments in both cases. However, the coronary atherosclerosis grade and extent in case 1 were more serious than those in case 2 . All of the examined coronary segments in case 1 revealed atherosclerotic changes of the intima, ranging from lesions classifiable as atherosclerotic tunica intima to secondary affection tunica intima, and stenosis from $51-100 \%$. In addition, atherosclerotic changes of normal tunica intima, fatty streak tunica intima, fibrous plaques tunica intima and atherosclerotic tunica intima were observed in the coronary segments of case 2, with coronary stenosis from $0-75 \%$.

Global analysis of coronary artery proteomics identifies proteins. The results of the global analysis of coronary artery proteomics are presented in Table II. Based on strict quality control and procedure criteria, a definite total of 515,061 spectrums, 21,703 peptides and 2,135 proteins were identified in the 20 coronary artery segments samples with repeated items removed, including 236,069 spectrums, 15,719 peptides and 1,835 proteins present in the 10 coronary artery segment samples of case 1 , and 278,992 spectrums, 18,362 peptides 
Table I. Natural history and histological classification of atherosclerotic lesions of the coronary artery samples.

A, Case 1

\begin{tabular}{lcccccccccccc}
\hline & Age (years) & Sex & LM & LAD-P & LAD-M & LAD-D & LCX-P & LCX-M & LCX-D & RCA-P & RCA-M & RCA-D \\
\hline Grade & 64 & Male & 3 & 4 & 3 & 4 & 4 & 4 & 3 & 4 & 4 \\
Stage & & & 4 & 3 & 3 & 3 & 4 & 3 & 3 & 4 & 3 & 3 \\
\hline
\end{tabular}

B, Case 2

\begin{tabular}{lcccccccccccc}
\hline & Age (years) & Sex & LM & LAD-P & LAD-M & LAD-D & LCX-P & LCX-M & LCX-D & RCA-P & RCA-M & RCA-D \\
\hline Grade & 69 & Male & 3 & 3 & 3 & 1 & 1 & 1 & 1 & 1 & 3 \\
Stage & & 3 & 3 & 3 & 0 & 0 & 1 & 1 & 2 & 3 & 1 \\
\hline
\end{tabular}

LM, left main trunk; LAD-P, proximal segment of the left anterior descending; LAD-M, midsegment of the left anterior descending; LAD-D, distal segment of the left anterior descending; LCX-P, proximal segment of the left circumflex; LCX-M, midsegment of the left circumflex; LCX-D, distal segment of the left circumflex; RCA-P, proximal segment of the right coronary artery; RCA-M, midsegment of the right coronary artery; RCA-D, distal segment of the right coronary artery. The stenosis grade was classified as follows: 1 , stenosis from 0 to $25 \%$; 2 , stenosis from $26-50 \%$; 3 , stenosis from $51-75 \%$; 4 , stenosis from $76-100 \%$. Stage was classified as follows: 0 , normal tunica intima; 1 , fatty streak tunica intima; 2 , fibrous plaques tunica intima; 3 , atherosclerotic tunica intima; 4 , secondary affection tunica intima.

and 2,046 proteins present in the 10 coronary artery segment samples of case 2 .

Characteristics distribution of the identified results. The distributions of precursors mass $\delta$, peptide length and number of unique peptides of identified proteins (Fig. 4) were employed to estimate the accuracy and reliability of the identification results. The mass $\delta$ of peptide segment precursors is basically distributed in the range of $\pm 0.1 \mathrm{Da}$, and the identified results achieving high accuracy are presented in Fig. 4A. Furthermore, the length distribution of peptide segments (Fig. 4B) demonstrates that the length of the majority of the identified peptide segments is concentrated in the range of 8-22, which is consistent with the molecular weight range recognized by a mass spectrometer (400-1,200).

A unique peptide segment is defined as a peptide that exists in only one protein group. The existence of the corresponding protein can be determined by the existence of the unique peptide segment, and the distribution of the number of unique peptide segments in all proteins identified in the present study is presented in Fig. 4C.

Analysis of the association of protein expression abundance with the histological classification of atherosclerotic lesions of the coronary artery samples. In the present study, the identification and quantification of proteins were performed using MaxQuant software (10). The identification criteria of significantly different proteins were a fold change (FC) $\geq 1.5$ or FC $\leq 0.67$. The present study only considered identified proteins to be abundantly differentially expressed between coronary artery segment samples when they met the aforementioned criteria. Comparisons of the identification and quantification of proteins under study are presented in Table III where, in each group, the expression abundance between significantly different proteins for those identified and quantified is shown.
According to these criteria, 390 proteins were abundantly differentially expressed between the fatty streak tunica intima and normal tunica intima groups. Compared with the normal tunica intima group, 257 proteins were observed as downregulated in the fibrous plaques tunica intima group, while 156 were determined to be upregulated. When comparing the atherosclerotic tunica intima, secondary affection tunica intima groups with normal tunica intima groups, the number of dysregulated proteins was 344 and 414, respectively. The most significant proteins of the whole integrated data were obtained through the intersection of the aforementioned four comparison sets, and the intersection revealed that a total of 174 proteins, including 4 upregulated [P54687 (BCAT1_HUMAN), Q06033 (ITIH3_HUMAN), Q13576 (IQGA2_HUMAN), Q96IY4 (CBPB2_HUMAN)] and 164 downregulated proteins (excluding 6 proteins with inconsistent expression tendencies), were obtained after comparing the fatty streak tunica intima, fibrous plaques tunica intima, atherosclerotic tunica intima and secondary affection tunica intima groups with the normal tunica intima groups. A Venn diagram analyzed the results of the distribution of shared differentially expressed protein families among multiple sets of comparisons, as presented in Fig. 5.

GO,COG and KEGGannotation of the differentially expressed proteins. In order to further investigate the biological and functional properties of all differentially expressed proteins, the present study used the GO annotation, COG annotation and KEGG annotation to analyze the differentially expressed proteins. The annotation results are presented in Fig. 6.

GO annotation with respect to the 174 differentially expressed proteins in the coronary artery samples demonstrated that the cellular component of these proteins was predominantly derived from the organelle $(17.18 \%)$, cell $(17.18 \%)$, cell part $(17.08 \%)$ and organelle part $(14.93 \%)$ 

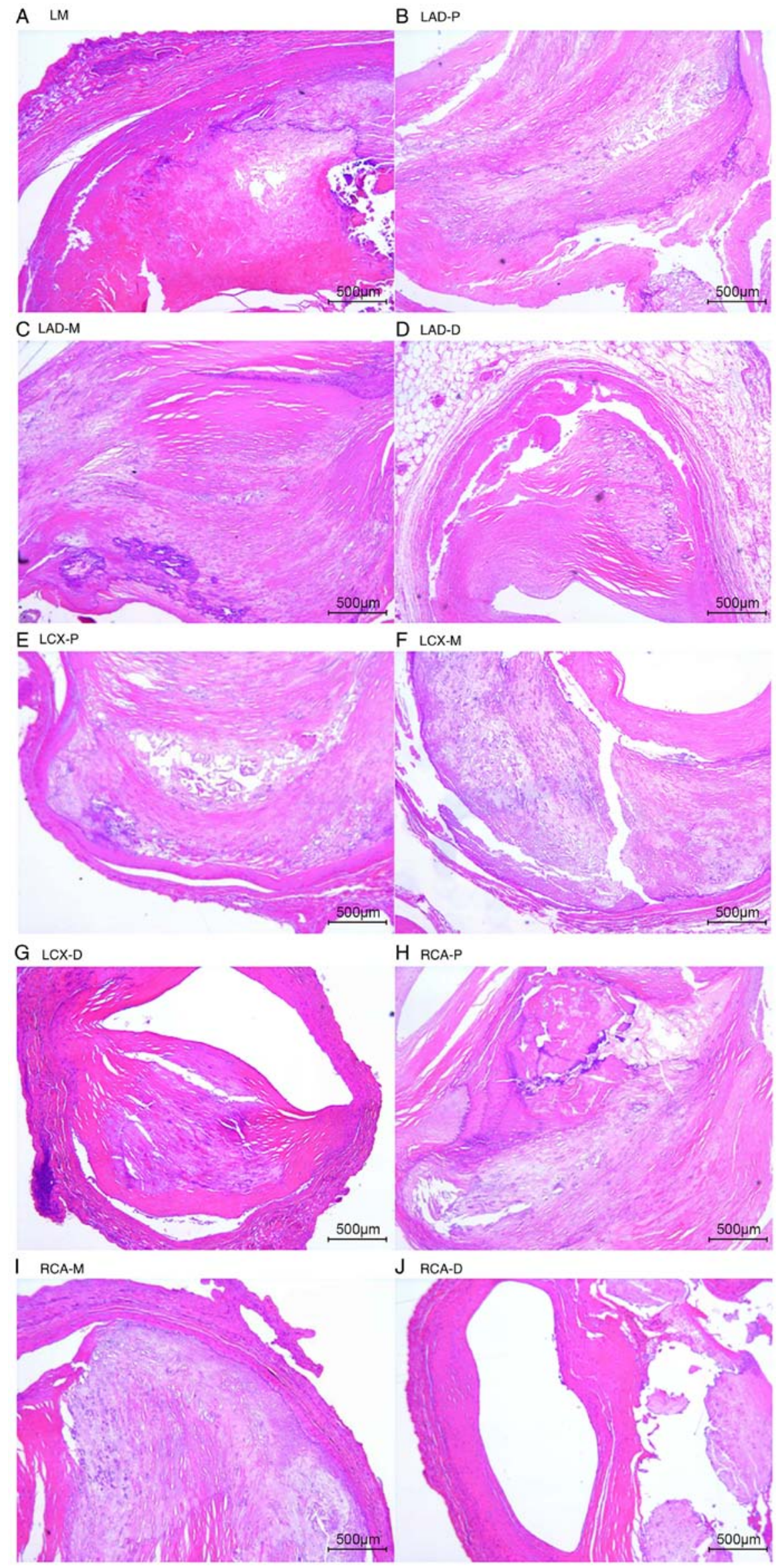

Figure 2. Histological characterization [panels (A-J)] of coronary arterial segments from case 1 stained by H\&E. H\&E staining results of the (A) left main trunk segment; (B) the proximal segment of the left anterior; (C) midsegment of the left anterior; (D) distal segment of the left anterior; (E) proximal segment of the left circumflex; (F) midsegment of the left circumflex; (G) distal segment of the left circumflex; (H) proximal segment of the right coronary artery; (I) left midsegment of the right coronary artery; (J) distal segment of the right coronary artery of case 1. H\&E, hematoxylin and eosin. Scale bar, $500 \mu \mathrm{m}$. 


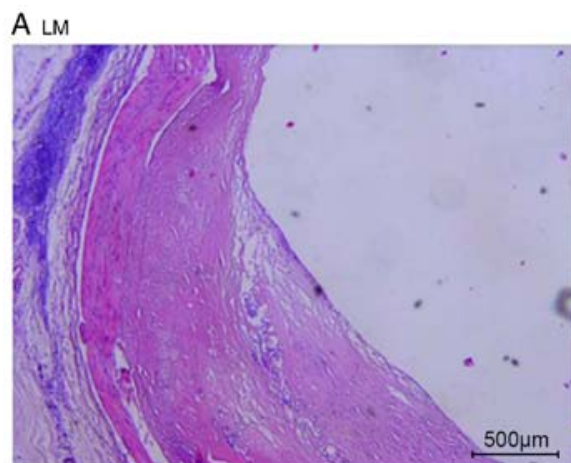

B LAD-P
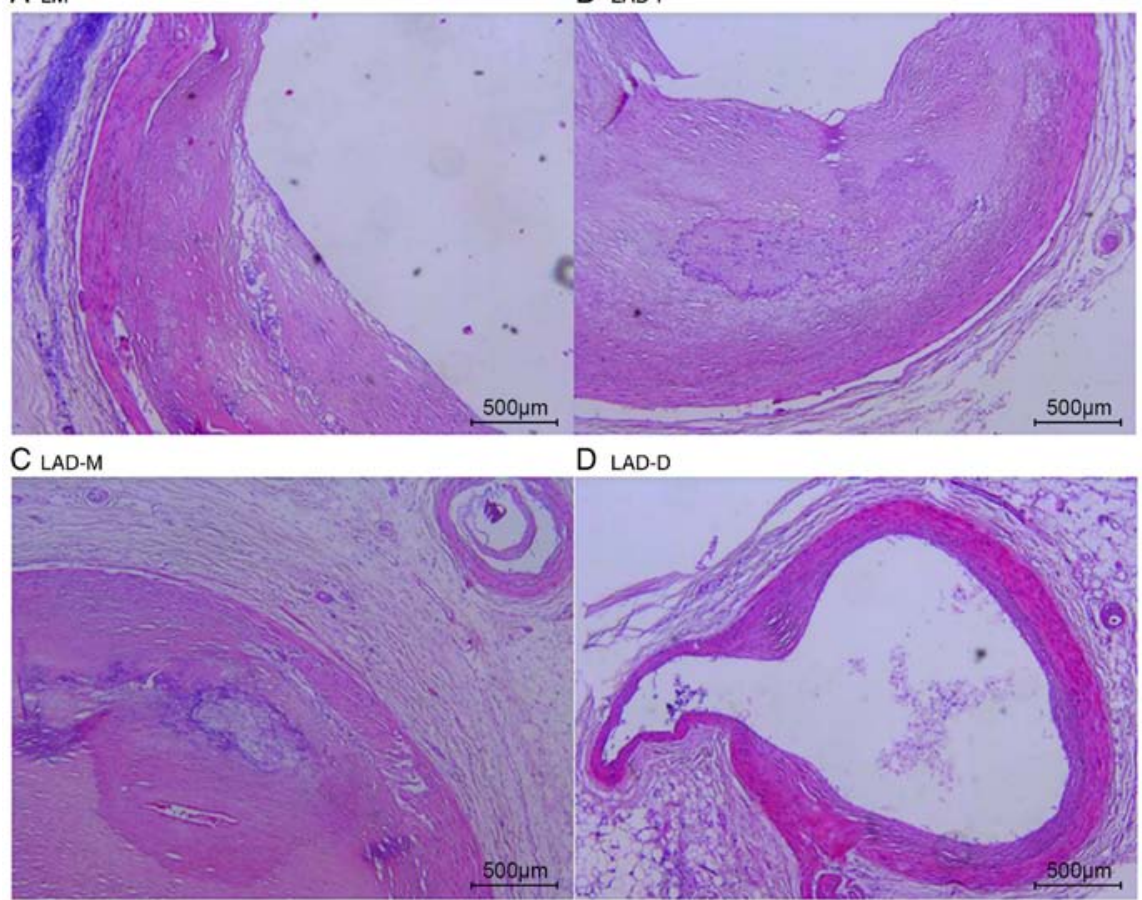

D LAD-D

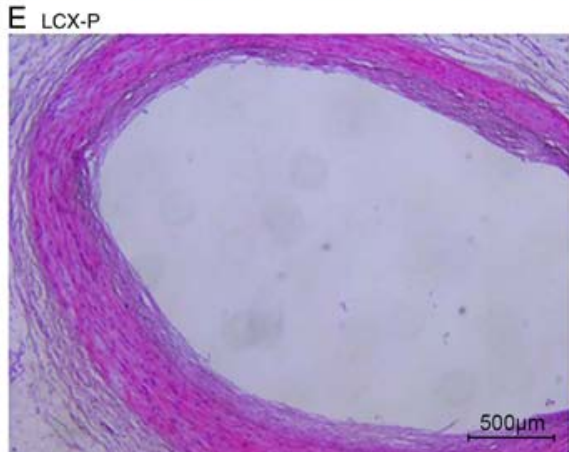

F LCX-M

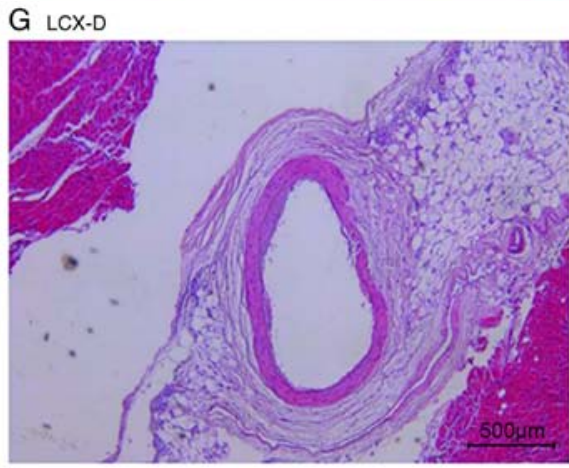

H RCA-P

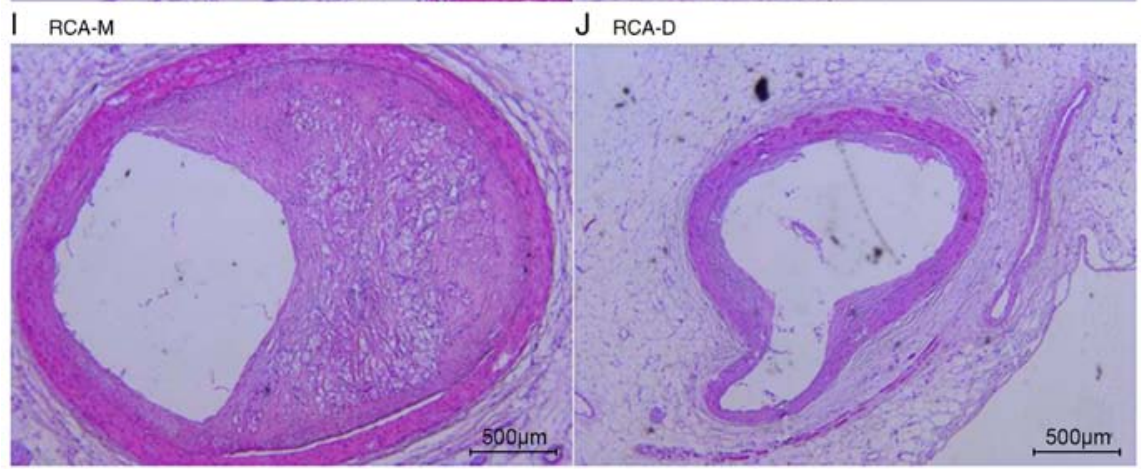

Figure 3. Histological characterization [panels (A-J)] of coronary arterial segments from case 2 stained by H\&E. H\&E staining results of the (A) left main trunk segment; (B) the proximal segment of the left anterior; (C) midsegment of the left anterior; (D) distal segment of the left anterior; (E) proximal segment of the left circumflex; (F) midsegment of the left circumflex; (G) distal segment of the left circumflex; (H) proximal segment of the right coronary artery; (I) left midsegment of the right coronary artery; (J) distal segment of the right coronary artery of case 2. H\&E, hematoxylin and eosin. Scale bar, $500 \mu \mathrm{m}$. 
Table II. Global analysis of coronary artery proteomics identifies proteins.

\begin{tabular}{lrrr}
\hline Type & Spectrum & Peptides & Proteins \\
\hline ALL_combined & 515,061 & 21,703 & 2,135 \\
Case 1 & 236,069 & 15,719 & 1,835 \\
Case 2 & 278,992 & 18,362 & 2,046 \\
LAD-D & 26,095 & 8,595 & 1,399 \\
LAD-D-2 & 25,976 & 7,831 & 1,279 \\
LAD-M & 23,247 & 7,940 & 1,227 \\
LAD-M-2 & 25,730 & 7,845 & 1,331 \\
LAD-P & 22,443 & 6,629 & 1,068 \\
LAD-P-2 & 28,156 & 8,638 & 1,357 \\
LCX-D & 19,319 & 6,523 & 969 \\
LCX-D-2 & 25,524 & 7,653 & 1,374 \\
LCX-M & 27,142 & 8,678 & 1,339 \\
LCX-M-2 & 26,934 & 7,943 & 1,404 \\
LCX-P & 21,642 & 7,814 & 1,197 \\
LCX-P-2 & 31,737 & 10,727 & 1,396 \\
LM & 25,468 & 8,073 & 1,294 \\
LM-2 & 27,092 & 8,323 & 1,328 \\
RCA-D & 22,635 & 7,537 & 1,123 \\
RCA-D-2 & 26,169 & 7,971 & 1,318 \\
RCA-M & 24,236 & 7,673 & 1,181 \\
RCA-M-2 & 31,845 & 10,615 & 1,486 \\
RCA-P & 23,842 & 7,494 & 1,257 \\
\hline
\end{tabular}

Some peptides were repeated in case 1 and 2. The total numbers of peptides and proteins presented in the table of 21,703 and 2,135 are with the repeated items removed. LM, the left main trunk; LAD-p, proximal segment of the left anterior descending; LAD-m, midsegment of the left anterior descending; LAD-d, distal segment of the left anterior descending; LCX-p, proximal segment of the left circumflex; LCX-m, midsegment of the left circumflex; LCX-d, distal segment of the left circumflex; RCA-p, proximal segment of the right coronary artery; RCA-m, midsegment of the right coronary artery; RCA-d, distal segment of the right coronary artery.

(Fig. 6A). Furthermore, the molecular function of these proteins primarily included binding $(41.47 \%)$, catalytic activity (33.24\%), transporter activity $(9.41 \%)$ and structural molecular activity (7.06\%) (Fig. 6B). In addition, the biological process that these proteins predominantly participated in included the cellular process $(16.95 \%)$, metabolic process $(15.99 \%)$, biological regulation (7.78\%) and multicellular organismal process (7.14\%) (Fig. 6C). The results demonstrated that these COG functions, including energy production and conversion, general function prediction only, lipid transport and metabolism, and cytoskeleton were primarily enriched in the differentially expressed proteins (Fig. 6D). KEGG pathway annotation indicated that these proteins were enriched in metabolic pathways (54.04\%), Huntington's disease (32.92\%), Parkinson's disease (32.3\%), oxidative phosphorylation (31.06\%) (Fig. S1), Alzheimer's disease (29.81\%) and microbial metabolism in diverse environments (15.53\%) signaling pathways (Table IV).
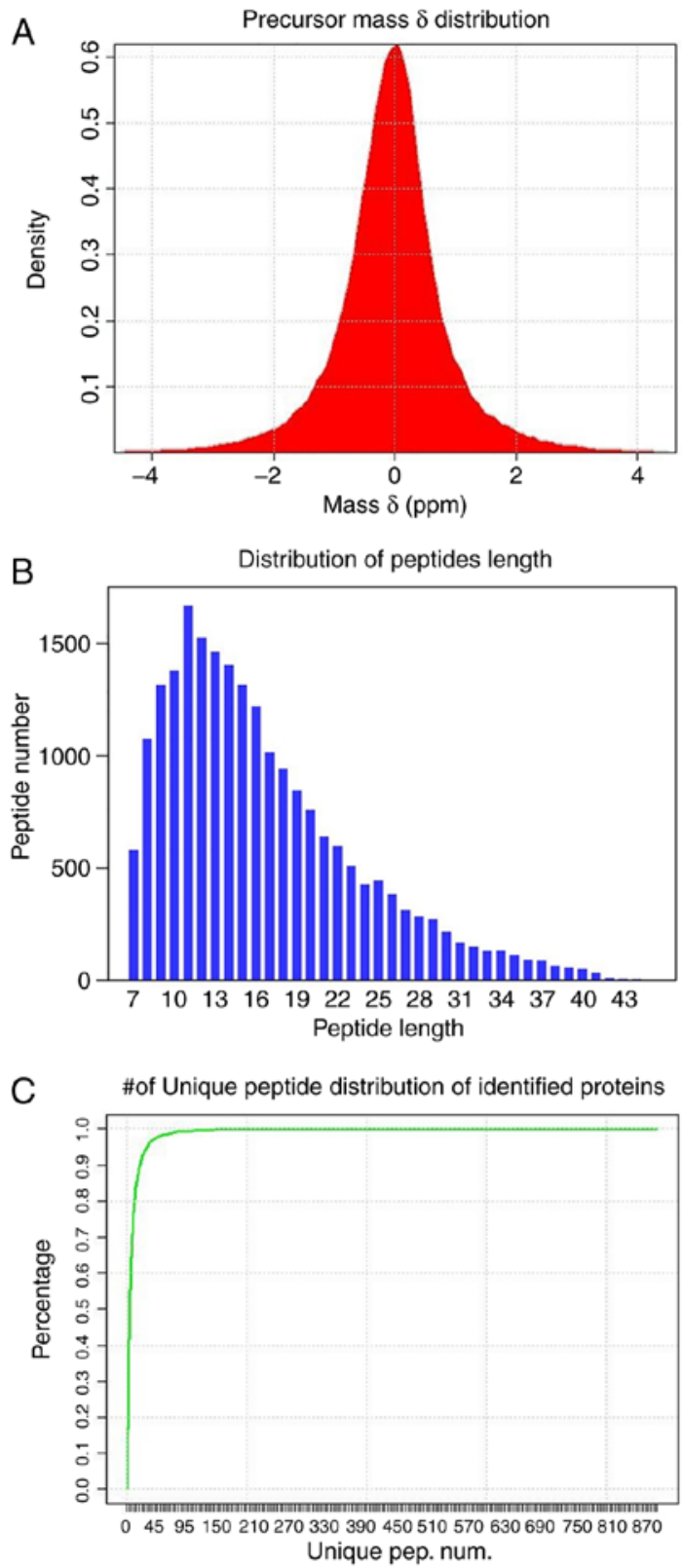

Figure 4. Characteristic distribution of the identification results. (A) The mass $\delta$ distribution of peptide segment precursors. (B) The distribution of the peptide segment lengths. (C) The distribution of the number of unique peptide segments for each identified protein.

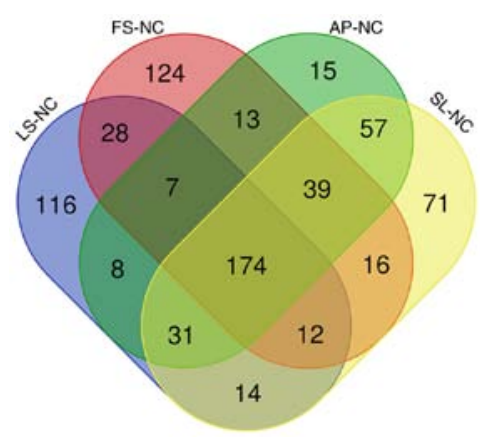

Figure 5. Venn diagram showing the distribution of the shared differentially expressed protein families. Venn diagram analyses of multiple group comparisons, including the secondary infection tunica intima group vs. normal tunica intima group; the atherosclerotic tunica intima group vs. normal tunica intima group; the fibrous plaque tunica intima group vs. normal tunica intima group; and fatty streak tunica intima group vs. normal tunica intima group. 
Table III. Analysis of protein expression abundance association with histological classification of atherosclerotic lesions of coronary artery.

\begin{tabular}{lccc}
\hline Type & sig. diff Num. & sig. UP num. & sig. DOWN num. \\
\hline Stage 1/Stage 0 & 390 & 174 & 216 \\
Stage 2/Stage 0 & 413 & 156 & 257 \\
Stage 3/Stage 0 & 344 & 120 & 224 \\
Stage 4/Stage 0 & 414 & 125 & 289 \\
Stage 4, 3, 2, 1/Stage 0 & 174 & - & - \\
\hline
\end{tabular}

Stage was classified as follows: 0 , normal tunica intima; 1 , fatty streak tunica intima; 2 , fibrous plaques tunica intima; 3 , atherosclerotic tunica intima; 4, secondary affection tunica intima. Quant Num, number of quantifications of protein group; diff Num., number of expression abundance significantly different proteins; UP num., number of expression abundance significantly upregulated proteins; DOWN num., number of expression abundance significantly downregulated proteins

Table IV. KEGG annotation of the differentially expressed proteins.

\begin{tabular}{lcc}
\hline Pathway & Different proteins with pathway annotation (n=161) (\%) & Pathway ID \\
\hline Metabolic pathways & $87(54.04)$ & ko01100 \\
Huntington's disease & $53(32.92)$ & ko05016 \\
Parkinson's disease & $52(32.3)$ & ko05012 \\
Oxidative phosphorylation & $50(31.06)$ & ko00190 \\
Alzheimer's disease & $48(29.81)$ & ko05010 \\
Microbial metabolism in diverse environments & $25(15.53)$ & ko01120 \\
Cardiac muscle contraction & $22(13.66)$ & ko00020 \\
Citrate cycle (TCA cycle) & $17(10.56)$ & ko05414 \\
Dilated cardiomyopathy & $16(9.94)$ & ko05410 \\
Hypertrophic cardiomyopathy & $16(9.94)$ & ko04510 \\
Focal adhesion & $11(6.83)$ & ko04810 \\
Regulation of actin cytoskeleton & $10(6.21)$ & ko00280 \\
Valine, leucine and isoleucine degradation & $10(6.21)$ & ko05412 \\
Arrhythmogenic right ventricular cardiomyopathy & $8(4.97)$ & ko04020 \\
Calcium signaling pathway & $7(4.35)$ & \\
\hline
\end{tabular}

GO and KEGG enrichment of the differentially expressed proteins. Cellular component analysis from the GO terms indicated that the differentially expressed proteins were mainly located in the mitochondrion, mitochondrial matrix, mitochondrial intermembrane, mitochondrial intermembrane space, mitochondrial inner membrane, mitochondrial outer membrane and mitochondrial respiratory chain complex I (Fig. S2). The GO terms for biological processes included respiratory electron transport chain, tricarboxylic acid cycle, mitochondrial electron transport NADH to ubiquinone and mitochondrial ATP synthesis coupled proton transport (Fig. S3). Several GO terms for molecular functions, including NADH dehydrogenase activity, NADH dehydrogenase (ubiquinone) activity, NADH dehydrogenase (quinone) activity, oxidoreductase activity acting on NADH or NADPH, and quinone or similar compound as acceptor were significantly enriched (Fig. S4). In addition, a KEGG pathway enrichment analysis was performed, which revealed that coronary artery disease-specific proteins were associated with
Parkinson's disease, oxidative phosphorylation, Huntington's disease, Alzheimer's disease, metabolic pathways, citrate cycle (TCA cycle), cardiac muscle contraction and microbial metabolism in diverse environments (Fig. 7). The GO function 'NADH dehydrogenase activity', the cellular component 'mitochondrion', and KEGG pathways 'metabolic pathways' enriched in the differentially expressed proteins, suggested that the mitochondrial energy metabolism may be underlying the occurrence and development of coronary artery atherosclerosis.

\section{Discussion}

The aim of the present study was to provide a comprehensive survey of human coronary artery proteins and identify individual proteins and potential regulatory pathways that differ between the histological classification of atherosclerotic lesions. The present study observed 2,135 proteins in the 20 coronary artery segments samples from two cases. Combined with the 


\section{cellular_component}

A

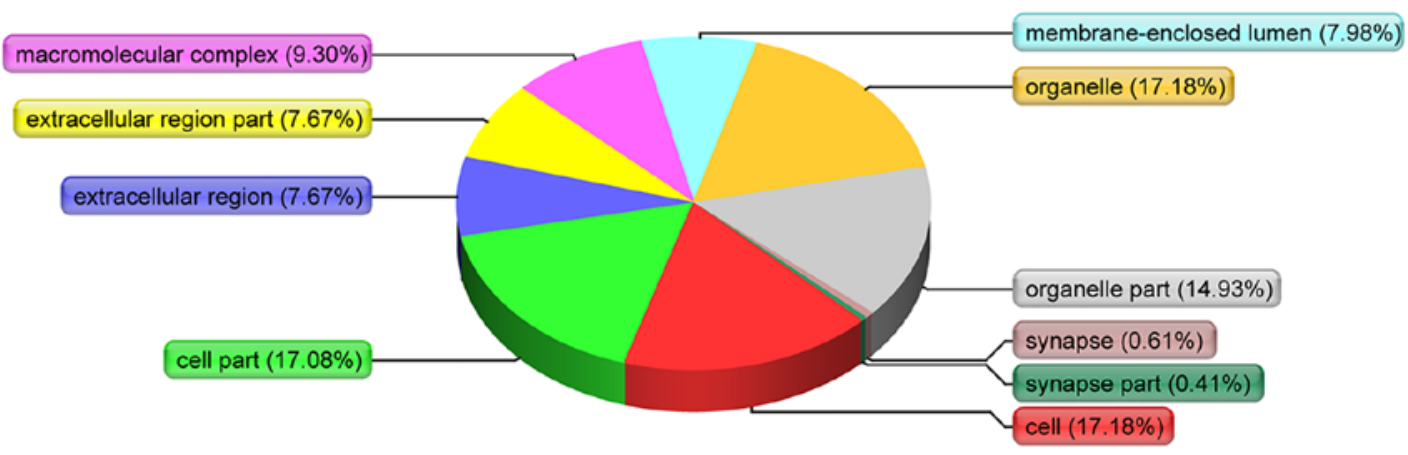

molecular_function

B

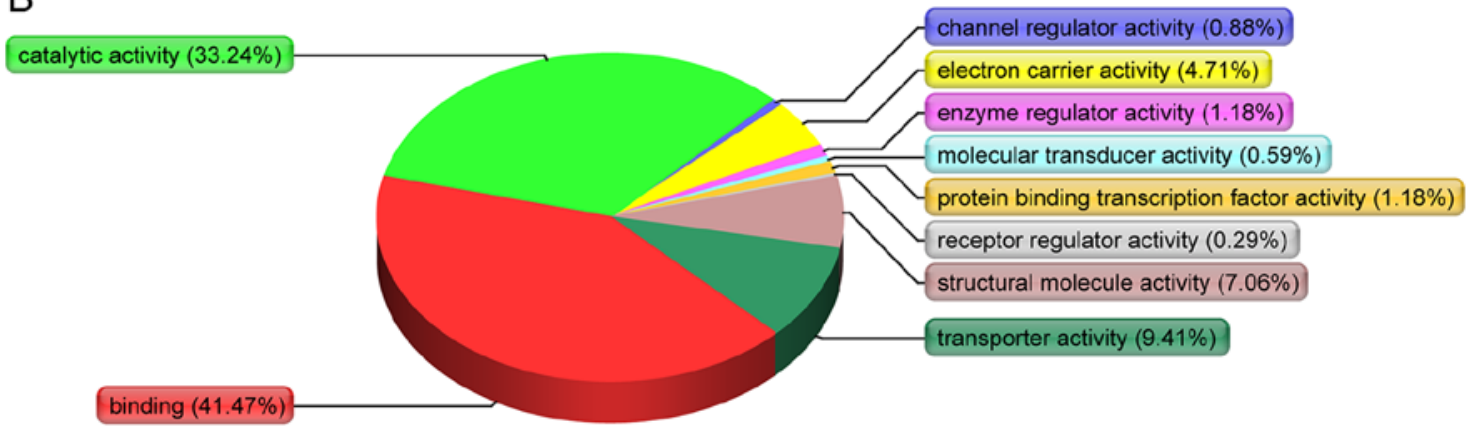

biological_process

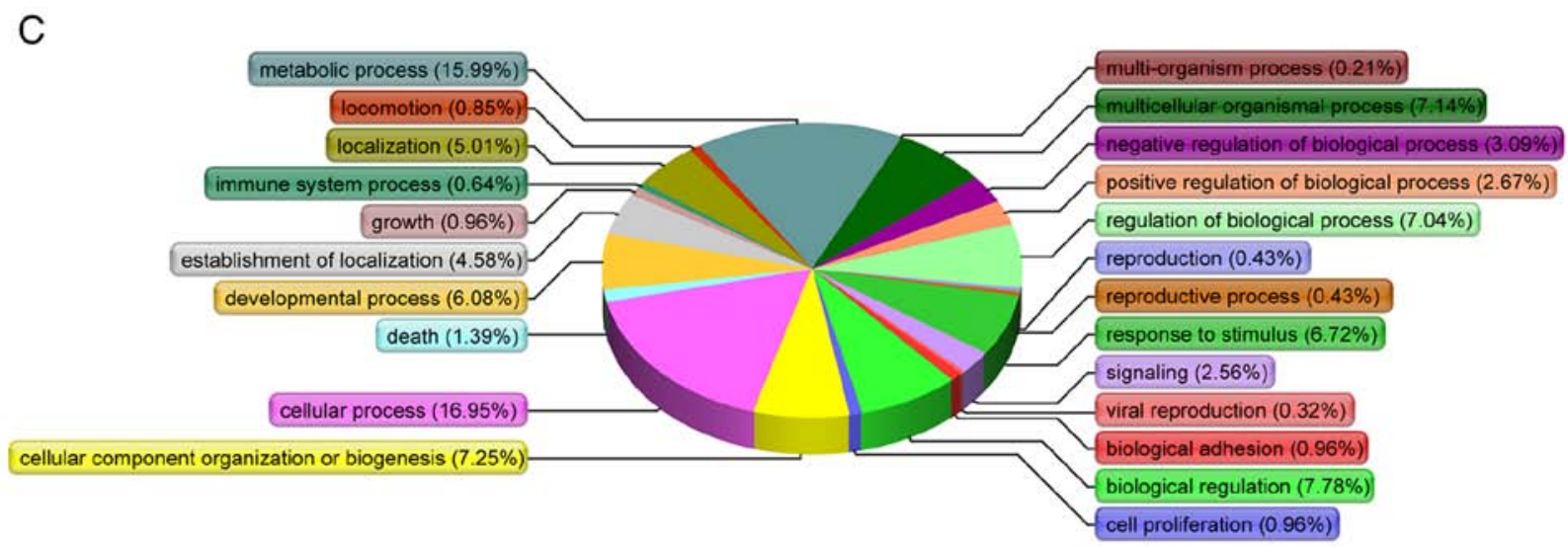

Figure 6. GO, COG and KEGG annotation of the differentially expressed proteins. (A) Cellular compartment annotation analysis: Organelle (17.18\%), cell (17.18\%), cell part (17.08\%) and organelle part (14.93\%); (B) molecular function annotation analysis: Binding (41.47\%), catalytic activity (33.24\%), transporter activity (9.41\%) and structural molecular activity (7.06\%); (C) biological process annotation analysis: Cellular process (16.95\%), metabolic process (15.99\%), biological regulation $(7.78 \%)$ and multicellular organismal process $(7.14 \%)$.

results of H\&E staining of the coronary artery samples, the intersection analysis suggested that the intersection reveals a total of 174 proteins, including 4 upregulated proteins and 164 downregulated proteins (excluding 6 proteins with inconsistent expression tendencies) that were obtained after comparing the fatty streak tunica intima, fibrous plaques tunica intima, atherosclerotic tunica intima and secondary affection tunica intima groups with the normal tunica intima groups. GO and KEGG enrichment analyses of the differentially expressed proteins revealed that the mitochondrial energy metabolism may be underlying the occurrence and development of coronary artery atherosclerosis. 


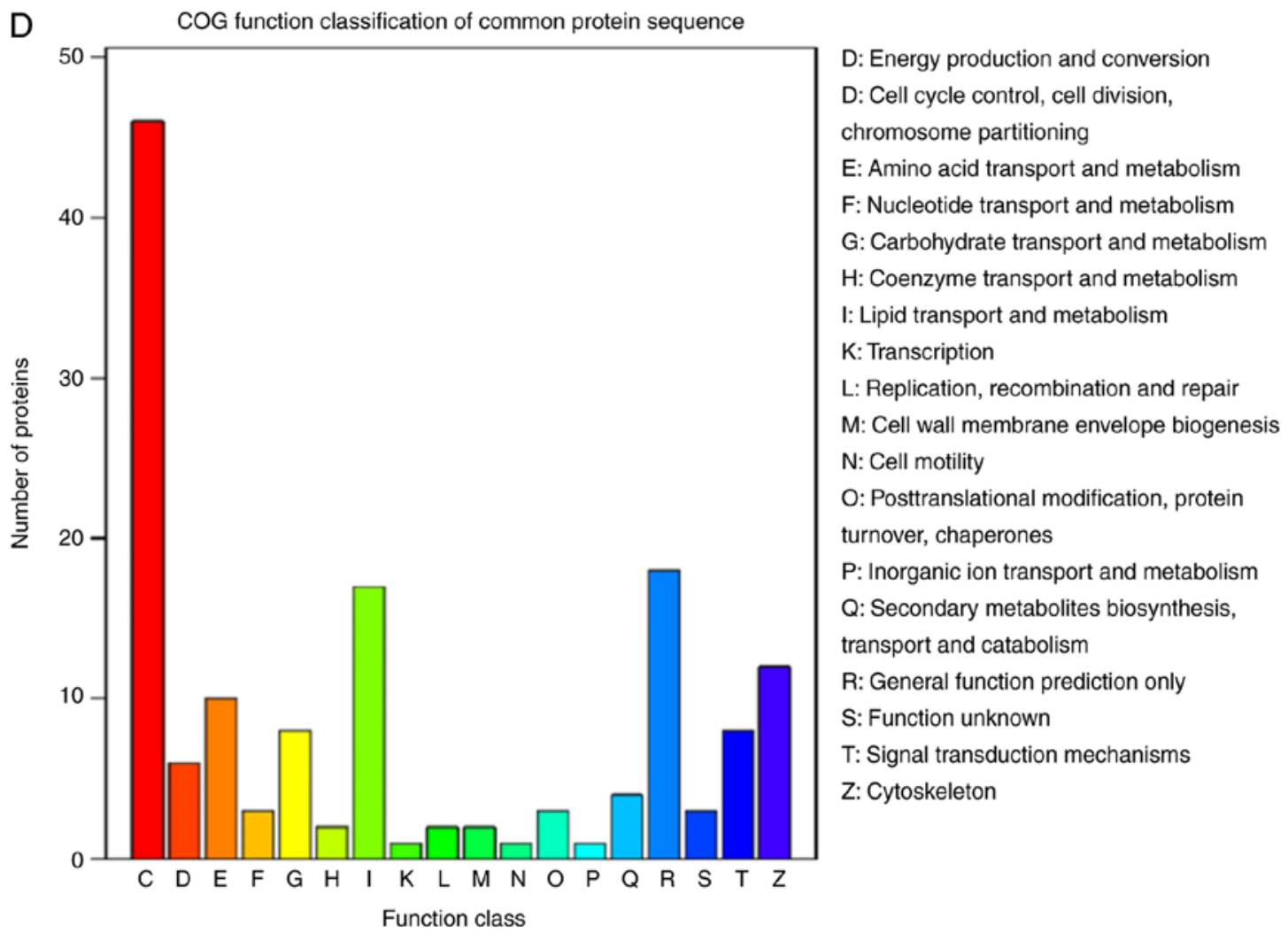

Figure 6. Continued. (D) COG functional annotation analysis: Energy production and conversion, general function prediction only, lipid transport and metabolism and cytoskeleton. GO, Gene Ontology; COG, Clusters of Orthologous Groups of proteins (COG); KEGG, Kyoto Encyclopedia of Genes and Genomes.

Propanoate metabolism
Butanoate metabolism
Dilated cardiomyopathy
Valine, leucine and isoleucine biosynthesis
Phenylalanine, tyrosine and tryptophan biosynthesis
Valine, leucine and isoleucine degradation
Hypertrophic cardiomyopathy (HCM)
Microbial metabolism in diverse environments
Cardiac muscle contraction
Citrate cycle (TCA cycle)
Metabolic pathways
Alzheimer's disease
Huntington's disease
Oxidative phosphorylation
Parkinson's disease

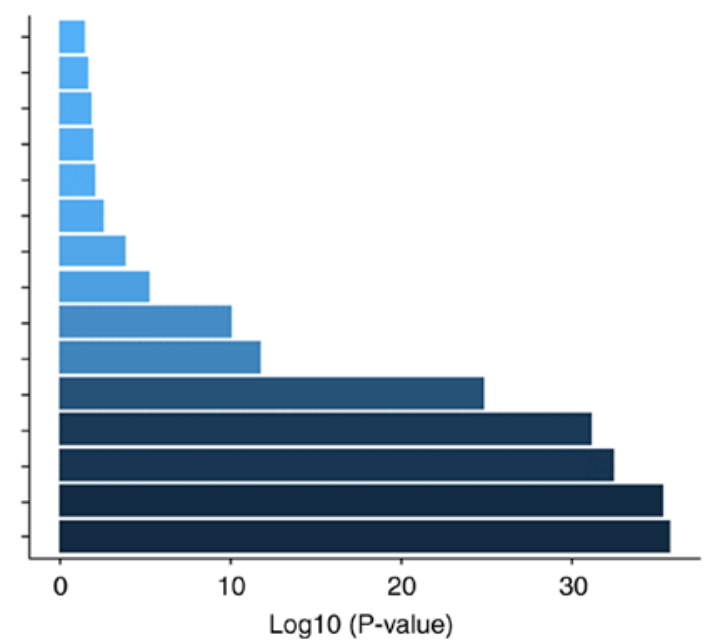

Figure 7. KEGG pathway enrichment analysis of the differentially expressed proteins. KEGG pathway enrichment analysis was performed, which revealed that coronary artery disease-specific proteins were associated with Parkinson's disease, oxidative phosphorylation, Huntington's disease, Alzheimer's disease, metabolic pathways, citrate cycle (TCA cycle), cardiac muscle contraction and microbial metabolism in diverse environments. KEGG, Kyoto Encyclopedia of Genes and Genomes.

Proteomics is defined as the comprehensive analysis of all proteins expressed in cells, tissues or organisms for the identification, quantification, post-translational modification, subcellular localization, protein-protein interaction and enzymatic activities (11). The proteomics approaches are becoming commonly available for the study of increasingly complex diseases since the early 2000s (12-14). Although a few proteomics studies on coronary artery disease may reflect the pathophysiological changes in coronary artery disease and may be used for the clinical study of coronary artery disease, the majority of samples involved in the proteomics studies of coronary artery disease are urine (15), coronary artery endothelial cells (16) and plasma (17). A total of 806 proteins were previously identified from 35 human coronary atherosclerotic samples by means of direct tissue proteomics analysis in a previous study (7); however, these samples were obtained in paraffin or frozen blocks. Another mass spectrometry analysis of coronary artery specimens from 100 
autopsied young adults presented the human coronary arterial proteome and proteomic features strongly associated with early atherosclerosis (6); however, these samples were only obtained from the LAD artery. The present study can be distinguished from previous investigations as the sampling of human coronary artery segments were underlying the majority of coronary artery disease morbidity and mortality, the quantity, quality and variety of proteins identified, and the virtue that the proteins were identified from two cases of comprehensive coronary artery tree samples where the effects of local anatomy context and natural history and histological classification of atherosclerotic lesions are manifested by $\mathrm{H} \& \mathrm{E}$ staining.

The human mitochondrial DNA (mtDNA) is a double-stranded, circular molecule of $16,569 \mathrm{bp}$ and contains 37 genes coding for proteins of the electron transport chain (ETC) [13 subunits of complexes I, III, IV and the ATP synthase (complex V)] that are essential for oxidative phosphorylation (OXPHOS), two rRNAs, 22 tRNAs and 13 polypeptides that are essential for normal mitochondrial function (18). Oxygen-consuming mitochondrial OXPHOS is the physiological means of ATP production in the heart. As a major source of cellular energy in the heart, mitochondria can produce ATP via the electron transport chain (ETC). Mitochondrial dysfunction can result in impaired cellular function, and has been associated with coronary artery disease. Results from a recent study have suggested that the transformation of mitochondrial metabolism from oxidative phosphorylation to glycolysis may be associated with coronary artery disease (19). In the present study, the analysis results indicated that a total of 174 proteins, including 4 upregulated proteins and 164 downregulated proteins, (excluding 6 proteins with inconsistent expression tendencies) were significantly associated with coronary artery disease. In addition, GO terms for molecular functions, including NADH dehydrogenase activity, NADH dehydrogenase (ubiquinone) activity, NADH dehydrogenase (quinone) activity, oxidoreductase activity acting on NADH or NADPH, the cellular component "mitochondrion, mitochondrial matrix, mitochondrial intermembrane, mitochondrial intermembrane space, mitochondrial inner membrane, mitochondrial outer membrane, and mitochondrial respiratory chain complex I', and KEGG pathways 'oxidative phosphorylation, metabolic pathways, citrate cycle (TCA cycle)' enriched in the differentially expressed proteins, suggested that the dysfunction of mitochondrial energy metabolism may be underlying the occurrence and development of coronary artery atherosclerosis. The results of the present study coincide with those of previous studies where it has been demonstrated that the altered mitochondrial dynamics and mitochondrial dysfunction are also key features of coronary artery disease $(20,21)$, and may shed light on the current understanding of the molecular mechanism of coronary artery disease with respect to environmental and genetic risk factors. From the present study, it can be concluded that the prevention of mitochondrial dysfunction may be a promising target in coronary artery disease. The present study demonstrated that it is possible to reverse the mitochondrial dysfunction, recover mitochondrial dynamics via retrieving the mitochondrial-associated protein expression, leading to decreased atherosclerosis of coronary artery.
The present study had several limitations. First, the human coronary artery samples of the present study were obtained from two cases with 20 segments; the sample size was too small to minimize the experimental bias; thus, a large-sample study is required in the future in order to validate the results obtained in the present study. Secondly, in the present study, the human coronary artery samples were selected postmortem; therefore, the potential effects of death on the stability of identified proteins, associated cellular functional processes, protein-protein interaction, protein networks and pathways should be noted. These limitations, however, do not withdraw from the accuracy and reliability of the identification results achieved by means of the analysis of the distributions of precursors mass $\delta$, peptide length and number of unique peptides of identified proteins. Thirdly, due to the functional validation assays not having been included in the present study, the specific mechanisms through which differentially expressed proteins may be involved in atherosclerosis development have not been established. In order to achieve the potential clinical utility of the identified proteins as coronary artery disease biomarkers, the functional role and mechanistic underpinning of the proteins, protein networks and pathways identified in the present study are required in future studies.

In conclusion, the present study identified 2,135 proteins in human coronary artery samples. Combined with the results of histological classification of the coronary artery samples, a total of 174 proteins, including 4 upregulated proteins and 164 downregulated proteins (excluding 6 proteins with inconsistent expression tendencies) were identified that were associated with coronary artery disease. In addition, GO and KEGG enrichment of the differentially expressed proteins revealed that the mitochondrial energy metabolism may be underlying the occurrence and development of coronary artery atherosclerosis.

\section{Acknowledgements}

Dr En-Zhi Jia is an Assistant Fellow at the Collaborative Innovation Center for Cardiovascular Disease Translational Medicine.

\section{Funding}

The present study was supported by the National Natural Science Foundations of China (grant nos. 81970302, 81170180, 30400173 and 30971257) and the Priority Academic Program Development of Jiangsu Higher Education Institutions.

\section{Availability of data and materials}

All data generated or analyzed during this study are included in this published article.

\section{Authors' contributions}

YaZ performed the collection of coronary artery samples and contributed to the writing and revising of the manuscript. JY performed the protein extraction and was involved in the writing of the manuscript. YF, FA and JC performed the LC-MS/MS analysis. YoZ, JJ, MG, ZM and HS performed 
the coronary artery segment preparation and the pathological analysis. QJ, CZ, MJ, JZ and GX performed the processing of mass spectrometry spectra and the bioinformatics analysis. EJ conceived and designed the study and contributed to the writing of the manuscript. All authors have read and approved the final manuscript.

\section{Ethics approval and consent to participate}

The methods were performed in accordance with the approved guidelines, and all experimental protocols were approved by the Ethics Committee of Nanjing Medical University and the First Affiliated Hospital of Nanjing Medical University. Informed consent from the bereaved family was obtained for the research use of the samples.

\section{Patient consent for publication}

Not applicable.

\section{Competing interests}

The authors declare that they have no competing interests.

\section{References}

1. Lobingier BT, Hüttenhain R, Eichel K, Miller KB, Ting AY, von Zastrow M and Krogan NJ. An Approach to spatiotemporally resolve protein interaction networks in living cells. Cell 169: 350-360.e12, 2017.

2. Thul PJ, Åkesson L, Wiking M, Mahdessian D, Geladaki A, Ait Blal H, Alm T, Asplund A, Björk L, Breckels LM, et al: A subcellular map of the human proteome. Science 356: eaal3321, 2017.

3. Van Rooden EJ, Florea BI, Deng H, Baggelaar MP, van Esbroeck ACM, Zhou J, Overkleeft HS and van der Stelt M: Mapping in vivo target interaction profiles of covalent inhibitors using chemical proteomics with label-free quantification. Nat Protoc 13: 752-767, 2018

4. Li KS, Shi L and Gross ML: Mass spectrometry-based fast photochemical oxidation of proteins (FPOP) for higher order structure characterization. Acc Chem Res 51: 736-744, 2018.

5. Distler U, Kuharev J, Navarro P and Tenzer S: Label-free quantification in ion mobility-enhanced data-independent acquisition proteomics. Nat Protoc 11: 795-812, 2016.

6. Herrington DM, Mao C, Parker SJ, Fu Z, Yu G, Chen L, Venkatraman V, Fu Y, Wang Y, Howard TD, et al: Proteomic architecture of human coronary and aortic atherosclerosis. Circulation 137: 2741-2756, 2018.

7. Bagnato C, Thumar J, Mayya V, Hwang SI, Zebroski H, Claffey KP, Haudenschild C, Eng JK, Lundgren DH and Han DK Proteomics analysis of human coronary atherosclerotic plaque: A feasibility study of direct tissue proteomics by liquid chromatography and tandem mass spectrometry. Mol Cell Proteomics 6 : 1088-1102, 2007.
8. Stary HC: Natural history and histological classification of atherosclerotic lesions: An update. Arterioscler Thromb Vasc Biol 20: 1177-1178, 2000.

9. Fernández-Coto DL, Gil J, Hernández A, Herrera-Goepfert R, Castro-Romero I, Hernández-Márquez E, Arenas-Linares AS, Calderon-Sosa VT, Sanchez-Aleman MÁ, Mendez-Tenorio A, et al: Quantitative proteomics reveals proteins involved in the progression from non-cancerous lesions to gastric cancer. J Proteomics 186: 15-27, 2018.

10. Tyanova S, Temu T and Cox J: The MaxQuant computational platform for mass spectrometry-based shotgun proteomics. Nat Protoc 11: 2301-2319, 2016.

11. Aebersold R and Mann M: Mass spectrometry-based proteomics. Nature 422: 198-207, 2003.

12. Florens L, Washburn MP, Raine JD, Anthony RM, Grainger M, Haynes JD, Moch JK, Muster N, Sacci JB, Tabb DL, et al: A proteomic view of the Plasmodium falciparum life cycle. Nature 419: 520-526, 2002.

13. Koller A, Washburn MP, Lange BM, Andon NL, Deciu C, Haynes PA, Hays L, Schieltz D, Ulaszek R, Wei J, et al: Proteomic survey of metabolic pathways in rice. Proc Natl Acad Sci USA 99: 11969-11974, 2002.

14. Washburn MP, Wolters D and Yates JR III: Large-scale analysis of the yeast proteome by multidimensional protein identification technology. Nat Biotechnol 19: 242-247, 2001.

15. Sun H, Wang D, Liu D, Guo Z, Shao C, Sun W and Zeng Y: Differential urinary proteins to diagnose coronary heart disease based on iTRAQ quantitative proteomics. Anal Bioanal Chem 411: 2273-2282, 2019.

16. Li SM, Liu WT, Yang F, Yi QJ, Zhang S and Jia HL: Phosphorylated proteomics analysis of human coronary artery endothelial cells stimulated by Kawasaki disease patients serum. BMC Cardiovasc Disord 19: 21, 2019.

17. Anwar MA, Dai DL, Wilson-McManus J, Smith D, Francis GA, Borchers CH, McManus BM, Hill JS and Cohen Freue GV: Multiplexed LC-ESI-MRM-MS-based assay for identification of coronary artery disease biomarkers in human plasma. Proteomics Clin Appl 11: e1700111, 2019.

18. Taanman JW: The mitochondrial genome: Structure, transcription, translation and replication. Biochim Biophys Acta 1410: 103-123, 1999.

19. Ait-Aissa K, Blaszak SC, Beutner G, Tsaih SW, Morgan G, Santos JH, Flister MJ, Joyce DL, Camara AKS, Gutterman DD, et al: Mitochondrial oxidative phosphorylation defect in the heart of subjects with coronary artery disease. Sci Rep 9: 7623, 2019.

20. Mercer JR, Cheng KK, Figg N, Gorenne I, Mahmoudi M, Griffin J, Vidal-Puig A, Logan A, Murphy MP and Bennett M: DNA damage links mitochondrial dysfunction to atherosclerosis and the metabolic syndrome. Circ Res 107: 1021-1031, 2010.

21. Sergin I, Evans TD, Zhang X, Bhattacharya S, Stokes CJ, Song E, Ali S, Dehestani B, Holloway KB, Micevych PS, et al: Exploiting macrophage autophagy-lysosomal biogenesis as a therapy for atherosclerosis. Nat Commun 8: 15750, 2017.

This work is licensed under a Creative Commons Attribution-NonCommercial-NoDerivatives 4.0 International (CC BY-NC-ND 4.0) License. 